\title{
Comentario. Arqueología al pensamiento de Bowman, repensar la Geografia y la Geopolítica desde y hacia el Perú
}

\section{Commentary. Archeology to Bowman's thought, rethinking Geography and Geopolitics from and to Peru}

\section{Comentário. Arqueologia para o pensamento de Bowman, repensando a Geografia e a Geopolítica de e para o Peru}

\author{
Juan Manuel Delgado Estrada \\ Universidad Nacional Mayor de San Marcos/ Universidad de Barcelona/CLACSO/UGI \\ https://orcid.org/0000-0002-2770-082X \\ juan.delgado10@unmsm.edu.pe \\ Mario Olivas Villanera \\ Universidad Nacional Mayor de San Marcos \\ https://orcid.org/0000-0002-3836-6679 \\ mario.olivas@unmsm.edu.pe
}

\begin{abstract}
RESUMEN
Cuando nos propusimos realizar el primer trabajo de la sección de clásicos de esta revista sobre Isaiah Bowman, no solo encontramos su producción sobre geografía política y geopolítica sino también sobre geografía física, y mucho sobre el Perú en sus territorios sureños que desconocíamos. Decidimos conocer más a Isaiah Bowman no solo como académico sino también como político y asesor de las altas esferas del poder norteamericano en las dos guerras mundiales, Bowman era un convencido que representaba al mundo libre y por eso se enfrentó en sus textos a la tristemente escuela geopolítica alemana, pero también en el Perú en sus trabajos describe la opresión de los indios andinos y amazónicos que eran explotados en regímenes casi de esclavitud en el sur del Perú, lo cuales detallo y fue un gran aporte para conocer la realidad del Perú. Pero en esa arqueología del pensamiento geográfico de Bowman también encontramos como el gran arquitecto de los estudios latinoamericanos desde las organizaciones académicos y políticas creadas para estudiar al sujeto social que se encuentra al sur del Río Bravo en México o Río Grande en Estados Unidos. Un artefacto de ese momento hegemónico del siglo XX desde Washington es el proyecto del Mapa al Millonésimo de Hispano America, que dio inicio al uso de nuevas técnicas cartográficas para recolectar geoinformación para ese propósito.
\end{abstract}

\begin{abstract}
When we set out to carry out the first work of the classics section of this magazine on Isaiah Bowman, we not only found his production on political and geopolitical geography but also on physical geography, and much about Peru in its southern territories that we did not know. We decided to get to know Isaiah Bowman more not only as an academic but also as a politician and advisor to the highest echelons of American power in the two world wars, Bowman was convinced that he represented the free world and that is why he sadly confronted the school in his texts German geopolitics, but also in Peru in his works he describes the oppression of the Andean and Amazonian Indians who were exploited in almost slavery regimes in the south of Peru, which he detailed and was a great contribution to know the reality of Peru.
\end{abstract}

Recibido:02/04/2021 - Aceptado: 24/06/2021 - Publicado: 25/09/2021

Citar como:

Delgado, J. y Olivas, M. (2021). Comentario. Arqueología al pensamiento de Bowman, repensar la Geografia y la Geopolítica desde y hacia el Perú. Espiral, revista de geografías y ciencias sociales, 3(5), 129-134. https://dx.doi.org/10.15381/espiral. v3i5.21184

C Los autores. Este artículo es publicado por Espiral, revista de geografías y ciencias sociales de la Universidad Nacional Mayor de San Marcos. Este es un artículo de acceso abierto, distribuido bajo los términos de la licencia Creative Commons Atribución 4.0 Internacional (CC BY 4.0) [https://creativecommons.org/licenses/by/4.0/deed.es] que permite el uso, distribución y reproducción en cualquier medio, siempre que la obra original sea debidamente citada de su fuente original. 
But in that archeology of Bowman's geographical thought we also find as the great architect of Latin American studies from the academic and political organizations created to study the social subject that is south of the Rio Bravo in Mexico or the Rio Grande in the United States. An artifact of that hegemonic moment of the 20th century from Washington is the project of the Map to the Millionth of Hispano America, which began the use of new cartographic techniques to collect geoinformation for that purpose.

\section{RESUMO}

Quando nos propusemos a realizar o primeiro trabalho da seção de clássicos desta revista sobre Isaiah Bowman, não encontramos sua produção apenas sobre geografia política e geopolítica, mas também sobre geografia física, e muito sobre o Peru em seus territórios do sul que não encontramos. saber. Decidimos saber mais sobre Isaiah Bowman não apenas como acadêmico, mas também como político e conselheiro dos mais altos escalões do poder americano nas duas guerras mundiais. Bowman estava convencido de que representava o mundo livre e por isso enfrentou tristemente o escola em seus textos geopolítica alemã, mas também no Peru em suas obras descreve a opressão dos índios andinos e amazônicos que foram explorados em regimes quase escravistas no sul do Peru, que detalhou e foi uma grande contribuição para conhecer a realidade de Peru. Mas nessa arqueologia do pensamento geográfico de Bowman também nos encontramos como o grande arquiteto dos estudos latino-americanos das organizações acadêmicas e políticas criadas para estudar a questão social que fica ao sul do Rio Bravo no México ou o Rio Grande nos Estados Unidos. Um artefato desse momento hegemônico do século 20 vindo de Washington é o projeto do Mapa ao Milionésimo da Hispano América, que deu início ao uso de novas técnicas cartográficas de coleta de geoinformação para esse fim.

PALABRAS CLAVES: Andes del Sur; Bowman; Geografía; Geopolítica; Perú.

KEYWORDS: Southern Andes; Bowman; Geography; Geopolitics; Peru.

PALAVRAS CHAVES: Southern Andes; Arqueiro; Geografia; Geopolítica; Peru.

\section{Las geografías arcaicas a las geografías históricas en los Andes Centrales}

El dominio territorial de los Andes Centrales es parte de esa migración que se inició cruzando la Beringia, primeramente, hacia Norteamérica, Centroamérica y finalmente se asentaron en los Andes Centrales de Sudamérica.

Estas sociedades arcaicas en esas circunstancias extremas dominaron el territorio y reprodujeron el código genético natural y el código social, donde finalmente la naturaleza humana se aleja de la naturaleza no humana, y ese es momento en donde la producción social del espacio de esos microterritorios, pasan a de ser la genesis de la Cosmovisión Andino - Amazónica de esa simbiosis Sociedad Naturaleza ${ }^{1}$.

El asentamiento de las sociedades arcaicas andinas - amazónicas en los andes centrales de Sudamérica, lo realizo en condiciones muy adversas, con una naturaleza no humana que lo sobrepasaba, que en algún momento "determinada" a esas sociedades arcaicas sus relaciones sociales con el territorio seleccionado donde desarrollo la racionalidad de la forma de trasmitir la acumulación de ese código socioespacial, es decir como un artefacto (arte + dato) y a la vez también como un artificio (arte + ingenio) $)^{2}$, es decir en una producción social de espacio material y simbólica entrelazada simbióticamente entre la geografía humana y la geografía física. Estas relaciones sociales con la naturaleza, manifiestan un habitus( Bourdieu, 1980 ) o prácticas culturales sobre el territorio, pero la naturaleza no humana sigue imponiéndose o determinando la forma de como asentarse en el borde costero, en los andes marítimos o los bosques húmedos orientales de los andes, pero los eventos extremos de origen físico en las diferentes alturas, latitudes y longitudes, los obligo a modificar sus relaciones con la naturaleza, por eso desarrollo una techne (Dussel, 2014: 3), que ayudo que esa acumulación de saberes en el sur de los andes centrales, sea sintetizado y resignificado de una forma material, como obras de infraestructura

1 El Hombre andino - amazónico creo un techne artesanal pero lógicamente científica y tecnológica para producir la dominación espacial del territorio de los andes centrales que era urgente para lograr su subsistencia, este fenómeno social es clave para que siglos después construya un artefacto para dominar la naturaleza humana de los andes centrales. 2 (Haesbaert [(2010), (2019)]) 
e ingeniería social para desarrollar su práctica o modelo de territorio, territorialidad y territorialización: reciprocidad y redistribución, que sería de alguna forma una cosmovisión y patrón de desarrollo territorial andino - amazónico, que luego tuvo un malencuentro (Etienne de La Boètie, [1576] 2016 ), cuando llegaron a desestructurar y deconstruir desde lo que hoy es Europa, e introdujeron esa modernidad/colonialidad, el cristianismo, el patriarcado, pero sobretodo modificaron la centralidad del ombligo del mundo andino - amazónico: Qosqo.

La palabra "Pirua" es runa simi como lo afirma el padre Blas Valera, luego fue mutando por su uso en "Pirú" y "Perú", siendo el significado más aceptado que trata de los continentes o recipiente de productos alimenticios múltiples que eran transportados y guardados, el profesor Porras Barrenechea nos dice que:

"El nombre del Perú, aplicado al Imperio de los Incas por los españoles, se difunde en el mundo a partir de 1534, después de la llegada de Hernando Pizarro a Sevilla y del desfile, ante la vista azorada de los habitantes y de los mercaderes genoveses y venecianos, del fabuloso tesoro de tinajas y barras de oro, a que se habían reducido los esplendorosos adornos del templo de Coricancha que sirvieron de irrisorio rescate al Inca Atahualpa" (Raúl Porras de Barrenechea, 1973).

La colonialidad/modernidad, el cristianismo, el patriarcado y el mercantilismo fueron trasladados de la metrópoli europea al nuevo Virreinato del Perú, desestructuraron y deconstruyeron la civilización originaria de los andes centrales, impusieron el eurocentrismo como una nueva territorialidad, desterritorialidad y multiterritorialidad (Haesbert), que bajo ese poder soberano y poder disciplinario (Foucault) de diversas escalas y territorios e híbridos: La primera globalización eurocentrada (Braudel), llego a lo que hoy es Perú, ese sistema - mundo (Wallerstein, 1987), donde se impuso normas, reglas y leyes en un territorio en red en el sentido global del lugar (Massey, 2000).

Bowman en su libro que explora, describe y analiza geográficamente el meridiano 73, previo trabajo de campo que lleva el mismo nombre "Los Andes del Sur Peruano" que se inició con la expedición de Hiram Bingham de la Universidad de Yale en 1911, en ese libro se sienta el estudio desde la geografía del sur peruano. La primera parte es sobre la geografía humana, y las relaciones de poder del sur del Perú, describe la posición hegemónica del gamonal frente al indio andino o amazónico pues también viajo por los bosques tropicales del oriente de los Andes, pero expuso la posición tan desventajosa para el indio en las ciudades, sugirió la división del trabajo a través de la raza ${ }^{3}$. En la segunda parte nos presenta la geografía física desde la mirada de la fisiografía, levantando la topografía, la cartografía y fotografías del territorio de estudio. Lo que nos llama la atención de ese libro es que describe que un grupo de indios andinos resolvieron ocupar el territorio intermedio entre los ricos valles agrícolas y las tierras de pastoreo que generalmente son altas, (Roosevelt, 1917).

\section{Bowman y los andes del sur del Perú}

Isaiah Bowman nació el 26 de diciembre del año 1878 en Ontario, Canadá, fue Bachiller en Ciencias de la Universidad de Harvard en 1902 y Doctor en la Universidad de Yale en 1908. Desde 1915 a 1935 fue presidente de la American Geographical Society, desde 1935 fue Recto de la Universidad de John Hopkins ${ }^{4}$.

3 Desde una perspectiva eurocéntrica, las relaciones de trabajo y capital con la idea de "raza" o "color" como se utiliza en Estados Unidos, esto se inició con la colonia, como un patrón de dominación moderno/colonial en La Colonialidad del poder en Latinoamerica y el Caribe (Quijano, 2013), pero el Perú se arrastra hasta la actualidad, y es Bowman quien en este libro donde evidencia que la realidad del Perú a inicios del siglo XX y casi en Centenario de la Independencia se utilice el trabajo no remunerado a través de la servidumbre, como una esclavitud moderna.

4 Isaiah Bowman, creo la carrera de geografía en Yale, además fue un gran representante de la geografía en EE. UU, pues fue asesor de los presidentes Wilson y Roosevelt durante las dos guerras mundiales. Fue discípulo del gran William Morris Davis, siendo este considerado el padre de la geomorfología, elegido el primer presidente de la Asociación Americana de Geógrafos (AAG) en 1904. 
En 1907 dirigió la expedición de la Universidad de Yale a Sudamérica, en 1911 viajo por el Perú como geógrafo de la expedición de la misma universidad en los Andes del Sur del Perú, apoyado por la American Geographical Society (AGS). La producción académica de Bowman sobre el Perú es amplia y conocida, entre ellas tenemos: Fisiografía de los Andes Centrales, 1909; Fisiografía de la Foresta (Bosques), 1911; Métodos de drenaje, 1911; Sud America, 1915; Los Andes del Sur del Perú, 1916; Los problemas del nuevo mundo en geografía política, $1921^{5}$.

Bowman en las expediciones de la Universidad de Yale del 1907 al 1913, reconoce los Andes Peruanos, recolectando mucha información, desde levantamiento topográfico que luego fue continuado por el Servicio Geográfico del Ejercito con un mapa topográfico 1:200,000 en la abrupta geografía física de los andes del sur peruano, libro del mismo título que Carlos Nicholson tradujo en Arequipa ${ }^{6}$, donde desde la geografía física nos sitúa todos los fenómenos físicos en ese territorio, desde digamos así como una historia geológica del sur del Perú, pero también recoge información de la geografía humana, con las contradicciones sociales de estas regiones andinos amazónicas, hasta llega a conocer una estación cauchera en bajo Urubamba7.

Bowman y Hiram Bingham ${ }^{8}$ fueron compañeros de las expediciones de Yale, estuvieron juntos en el "descubrimiento de Machu Pichu" y de cómo sin el auspicio de la National Geographic Society y la The Geographical Review que a la fecha es su revista institucional (Javier Flores, 2019), no se hubiera logrado esa expedición.

La ASG fue fundada en 1852, posteriormente se creó la Oficina de investigación de Hispano America, siendo su objetivo crear el Mapa Millonésimo que se inició en 1920 y que al año 1945 con una extensión de 107 páginas de cartografía de 20 países independientes y 14 colonias en nuestro continente.

\section{Arqueología del pensamiento de Bowman sobre el Perú y los Estados Unidos de America}

Bow manllego a ser un geógrafo muy importante para las relaciones internacional de los EE. UU no solo por los servicios diplomáticos a los gobiernos norteamericanos en las dos guerras mundiales, sino también porque a través de la AGS se iniciaron los Estudios Latinoamericanos desde los Estados Unidos de America, se inició la exploración, compilación y estudio de ese sujeto del sur del continente desde las instituciones supranacionales como la OEA y su Instituto Panamericano de Geografia e Historia - IPGH, organización que hace suya el proyecto del Mapa al Millonésimo de Hispano América en 1935.

Así como su maestro William Morris Davis quien, desde la geología, geomorfología y geografía junto a sus alumnos, entre ellos Bowman empiezan una "primavera del conocimiento geográfico" en las universidades norteamericanas, creándose escuelas, departamentos y centros de investigación en diversas universidades norteamericanas, aunque siempre se cuestionó si la geografía debería ser una disciplina universitaria (Smith, 1987:165).

\footnotetext{
5 En 1942 publico su obra más difundida “Geography vs. Geopolitics” en Geographical Review, este texto fue una respuesta desde la "geografía científica" anglosajona al pensamiento geopolítico alemán en plena segunda guerra mundial.

6 En los Andes del sur del Perú al ser traducido y al tener información muy relevante de geografía física y humana, tiene un valor extraordinario documentario y toponímico, pero también ya describe el problema de la tierra y sus distorsiones de la propiedad, del indio, del abuso y brutalidad al realizar trabajos que no eran remunerados, o de las mujeres que eran consideradas "presas legales".

7 Sobre los caucheros: "Que historia podría contar en un muelle de Nueva York, una bola de caucho, secada al humo, si pudiera hablar, del sendero anegado de la selva, de los peones esclavizados de los abusos viles de gente inmorales, ;de todo el trabajo y enfermedades que hacen de las tierras tropicales un infierno!"

8 En el centenario del descubrimiento de Machu Pichu en 1911 se discutió en foros académicos y políticos si era pertinente celebrar dicho "descubrimiento" pues existía un cuestionamiento a la Universidad de Yale sobre la devolución de piezas arqueológicas cedidas para su estudio. Además, que también se cuestionó de la forma de cómo se realizaron los estudios arqueológicos en el sitio y alrededores de Machu Pichu; ver El libro de Amy Cox Hall, Framing a Lost City. Science, Photography, and the Making of Machu Picchu.
} 
La Sociedad Geográfica de Lima era en la primera parte del siglo XX la institución del gobierno peruano que emite la cartografía oficial y que poco a poco introduce la necesidad de la formación disciplinar de la geografía en los claustros universitarios peruanos. Esta hegemonía dentro del aparato estatal es poco a poco reemplazada por el 1 Servicio Geográfico del Ejército.

La geografía peruana universitaria todavía se encontraba orientada a la geografía física clásica, no es hasta 1922 que se crea la obligatoriedad del curso de Geografia Social, en 1928 cambia de nombre a Geografía Humana del Perú en la UNMSM, no es hasta las décadas de los 40's que Javier Pulgar Vidal presenta su tesis en el IPGH revolucionando la tradición geográfica de estudiar el territorio peruano en costa, sierra y selva, para introducir las 8 regiones naturales del Perú presentada en la Tercera Asamblea del Instituto Panamericano de Geografía e Historia reunida en Lima en 1941(Córdova, 2011:182), mientras que Emilio Romero presenta su obra. Geografía económica del Perú (1930, luego reimpresa en 1936, 1953,1961 y una edición abreviada en 1968).

En esta arqueología del saber ${ }^{9}$ del pensamiento de Bowman, nos quedamos con el comentario de Marcos Cueto (1992) sobre la importancia de la traducción en 1938 del libro Los Andes del sur del Perú, realizado por Carlos Nicholson en Arequipa, que nos sitúa a inicios del proceso de desarrollo del siglo XX, pero también nos denota el legado de Bowman con el estudio del Perú y su influencia en el pensamiento geográfico contemporáneo y en las historias de las ideas de las ciencias sociales y físicas, con una ausencia de poder medir ese impacto hasta hoy.

Bowman, describiendo la realidad social del Perú dice: "Ambos bandos (gobiernistas y revolucionarios) explotan al indio hasta el último limite contra la ley y más allá de los límites de la decencia", nos da pistas de ese pensamiento desde el discurso y el análisis semántico de este discurso, pero fuera de las estructuras del saber oficial en varias de sus obras sobre el sur del Perú.

Es una tarea pendiente un análisis más exhaustivo de las obras de Bowman sobre el Perú, especialmente uno que ha sido poco mencionado que fue publicado en 1942, como es "The Ecuador-Peru Boundary Dispute" del año 1942, es decir se cree que tuvo mucha influencia en la firma del Protocolo de Paz, Amistad y Límites de Río de Janeiro, entre Perú y Ecuador sobre las fronteras entre ambos países.

\section{Referencias Bibliográficas}

Agulló, J. (2020). "Comentario. Releer a Bowman, repensar la Geopolítica". Geopolítica(s). Revista de estudios sobre espacio y poder, 11(2), 379-385.

Bowman, I. (1911) Forest physiography; physiography of the United States and principles of soils in relation to forestry. [New York, J. Wiley \& sons; etc., et] [Web.] Retrieved from the Library of Congress, https://lccn.loc.gov/11029383 .

Bowman, I. (1916) [1938]. The Andes of Southern Peru; Geographical Reconnaissance along the Seventy-Third Meridian, Published for The American Geographical Society of New York by Henry Holt and Company. Traducido por Carlos Nicholson. Editorial La Colmena S.A. Arequipa, Perú. 268 p.

Bowman, Isaiah. (1921). The New World; problems in political geography, World Book Company, Nueva York.

Bowman, I. (1924). Desert Trails of Atacama (American Geographical Society, New York, Special Publications No 5, 1924: 362 p.).

9 Quizás la arqueología y su análisis del discurso pudiera ser útil en algunos casos, pero con Bowman es casi imposible por la contrariedad del personaje que estudia y escribe sobre el Perú, pero muy diferente a cuando el modifica la escala de sus trabajos y lo ve de forma global desde la geopolítica, se aleja totalmente de ese microespacios estudiado como los andes centrales, es decir esa diferencia entre la totalidad y la singularidad de practica discursiva con un personaje como Bowman, pero como dice Foucault (1969) la arqueología es unidimensional y antirrepresentacionista, le interesa el discurso como tal (Kreimerman, 2014). 
Bowman, I. (1934). Geography in Relation to the Social Sciences (Report of the Commission on the Social Studies, American Historical Association., Part 5). New York: Charles Scribner's Sons.

Bowman, I. (1937). A New Chapter in Pan-American Cartography. En Melanges de geographie et d'orientalisme offerts a E.-F. Gautier (pp.88-95). Tours: Arrault et Cie.

Bowman, Isaiah. (1942). “The Ecuador-Peru Boundary Dispute”, Foreing Affairs, vol. 20, núm. 4, pp. 757-761.

Bowman, I. (2020). Geografía vs. Geopolítica. Geopolítica(s). Revista de estudios sobre espacio y poder, 11(2), 365-378.

Carter, G. (1950). Isaiah Bowman, 1878-1950. Annals of the Association of American Geographers, 40(4), 335-350. Retrieved August 9, 2021, from http://www.jstor.org/ stable/2561147

Department of State (1937) Actas de la segunda asamblea general del Instituto Panamericano de Geografia e Historia, celebrada en Washington del 14 al 19 de octubre de 1935. Conference Series $N^{\circ} 28$. United States, Gobernement Printig Office, Washington, 1937.

Foucault, M. (1969) [2008]. La Arqueología del Saber. Buenos Aires, Argentina: Siglo XXI.

Kreimerman, E. (2014). La arqueología del saber cómo método de investigación. IV Encuentro Latinoamericano de Metodología de las Ciencias Sociales, 27 al 29 de agosto de 2014, Heredia, Costa Rica. La investigación social ante desafíos transnacionales: procesos globales, problemáticas emergentes y perspectivas de integración regional. En Memoria Académica. Disponible en: http://www.memoria.fahce.unlp.edu.ar/trab_eventos/ ev.8214/ev.8214.pdf

Quijano, Aníbal (2013). El trabajo. Argumentos, 26 (72), 145-163. [Fecha de Consulta 21 de julio de 2021]. ISSN: 0187-5795. Disponible en: https://www.redalyc.org/articulo. oa?id $=59528835008$

Roosevelt, T. (1917). The Andes of Southern Peru. A Review. Geographical Review, 3(4), 317322. doi:10.2307/207432. 